Research Paper

\title{
An investigation of the distribution and location of mast cells affected by the stiffness of substrates as a mechanical niche
}

\author{
Hong-Wei Yang${ }^{1}$, Xin-Yue Liu ${ }^{2}$, Zhou-Feng Shen ${ }^{1}$, Wei Yao ${ }^{\bowtie}$, Xiao-Bo Gong ${ }^{2}$, Hua-Xiong Huang ${ }^{3}$, \\ Guang-Hong Ding 1 \\ 1. Department of Aeronautics and Astronautics, Fudan University, Shanghai 200433, China. \\ 2. Key Laboratory of Hydrodynamics (Ministry of Education), Department of Engineering Mechanics, Shanghai Jiao Tong University, Shanghai 200240, China. \\ 3. Department of Mathematics and Statistics, York University, Toronto, Ontario, Canada, M3J 1P3. \\ $\bowtie$ Corresponding authors: Wei Yao (weiyao@fudan.edu.cn) and Xiao-Bo Gong (x.gong@sjtu.edu.cn). \\ (c) Ivyspring International Publisher. This is an open access article distributed under the terms of the Creative Commons Attribution (CC BY-NC) license \\ (https://creativecommons.org/licenses/by-nc/4.0/). See http://ivyspring.com/terms for full terms and conditions.
}

Received: 2018.04.17; Accepted: 2018.06.04; Published: 2018.06.22

\begin{abstract}
The distribution and location of mast cells are closely related to their physiological and pathological functions, such as allergic responses, immunity, and fibrosis, and are used in acupuncture. In this study, the distribution of mast cells in vivo was observed, and mechanical clues for understanding their distribution based on mechanical niches were explored. By toluidine blue staining and immunohistochemical staining, we examined the distribution and location of mast cells in rat skin and found that mast cells are distributed in a spatially nonuniform manner, preferring to locate at regions in the tissue and extracellular matrix with stiffness changes. In vitro experiments for studying the distribution of rat basophilic leukemia (RBL-2H3) mast cell line on poly-di-methyl-siloxane (PDMS) substrates with stiffness variations were performed. It was found that RBL-2H3 cells migrate and tend to remain in the areas with stiffness variations. The present research suggests that changing the stiffness of local tissues may stimulate mast cell recruitment, which may be the method by which some traditional Chinese medicine treatments, such as acupuncture. On the basis of the origin of mast cells and our experimental results, we predict that mast cells exist in tissues that contain permeable capillaries and prefer regions with stiffness changes. We discussed this prediction using examples of specific tissues from some cases.
\end{abstract}

Key words: Mast cells; Stiffness variations; Distribution; Durotaxis; Acupuncture; Fibrosis

\section{Introduction}

Mast cells play important roles in allergic responses [1], immunity [2], angiogenesis [3], wound healing [4], acupuncture [5,6], and other processes. Although the biological and biochemical characteristics of mast cells in allergic inflammation have been intensively studied since they were first discovered by Paul Ehrlich in the late 1800s [7-10], the interaction of mast cells with their surrounding mechanical environment is still largely unknown. In recent decades, cell migration and mast cell functions have become two hot topics in the field of medical physiology, and both are difficult problems [11]. The biological behaviors of mast cells under different mechanical conditions and matrix environments have attracted significant attention $[7,12,13]$.

As we know, mast cells are distributed widely in the connective tissue of the skin, which consists of multicomponent functional tissues and extracellular matrix with different stiffness levels (Young's modulus) [14]. Interactions with extracellular matrix components appear to be important for the migration and location of mast cells in tissues [13]. The migration of mast cells always occurs following chemical activation or mechanical stimulation and plays an important role in regulating homeostasis. 
There are many findings about the location, distribution and recruitment of mast cells [15, 16]. Mast cells are found to be resident in tissues throughout the body, particularly in association with structures such as blood vessels and nerves and in proximity to surfaces that interface with the external environment [7]. These phenomena are still relatively independent, and there is no suggesting rule by which we understand the phenomena of their migration and distribution. Defining a general rule that explains the location and function of mast cells in physiological and pathological tissues would help to guide further research and aid in the treatment of tissue conditions related to mast cells.

In this study, the distribution and location of mast cells around the main tissues in rat skin were observed. It was found that mast cells are distributed in a spatially nonuniform manner, in that they prefer areas with stiffness variations in the tissue and extracellular matrix. To verify this unusual phenomenon in vitro, experiments on the distribution of rat basophilic leukemia (RBL-2H3) mast cell line on poly-di-methyl-siloxane (PDMS) substrates with stiffness variations were performed. It was observed that RBL-2H3 cells migrate and tend to be found in the areas with stiffness variations. Combined with the viability and concentration of RBL-2H3 on different PDMS substrates, we verified that RBL-2H3 cells tend to migrate to the areas with stiffness variations. On the basis of the origin of mast cells and our experimental results, we predicted that mast cells exist in tissue that contains permeable capillaries and that they should be located at areas with stiffness variations. This hypothesis was discussed in normal and pathological tissues. We further showed that traditional manual acupuncture could recruit and activate mast cells by lifting and twisting needles. The present result suggests that changing the stiffness of local tissues may stimulate mast cells to be recruited to sites of acupuncture treatment. The present studies on the migration, distribution, matrix circumstance and mechanical response of mast cells play a profound role in understanding the role of mast cells in physiological and pathological processes [17].

\section{Methods and materials}

\section{Animals}

The present study was performed in accordance with the guidelines of the Animal Care and Use Committee of Shanghai Research Center for Acupuncture and Meridians, which are based on the $\mathrm{NIH}^{\prime}$ s Guide for the Care and Use of Laboratory Animals. The experimental protocols were approved by the Animal Care and Use Committee of the Shanghai Research Center for Acupuncture and
Meridian. Male Sprague-Dawley (SD) rats (200 $\pm 20 \mathrm{~g})$ from the Shanghai Experimental Animal Center of the Chinese Academy of Science were housed in cages in a temperature-controlled environment $\left(22^{\circ} \mathrm{C}-25^{\circ} \mathrm{C}\right)$ with a 12/12-hour light/dark cycle. Food and water were made available ad libitum. All animals were handled with care to prevent infection and to minimize stress. Animals were chosen randomly to perform each group experiment.

\section{Cell culture}

RBL-2H3 mast cell lines were obtained from the Shanghai Science Academy cell bank (Shanghai, China). The cells were grown in minimal essential medium (MEM) with Earle's salts containing 15\% fetal bovine serum and 1\% L-glutamine, all these culture reagent were from Gibco (Invitrogen, Grand Island, NY, USA). Cells were cultured in an incubator with a humidified atmosphere $\left(5 \% \mathrm{CO}_{2}\right)$ at $37^{\circ} \mathrm{C}$ and were used in an exponential phase of growth. The initial cell concentration in each group of experiments was the same in every dish.

\section{Toluidine blue staining}

Tissue samples from the Zusanli point (ST36) were collected centrally with different sizes of skin biopsy punches (Electron Microscopy Sciences, Hatfield, PA, USA) according to the experimental requirements after decapitation of the animals under narcosis $(10 \%$ chloral hydrate $0.4 \mathrm{~mL} / 100 \mathrm{~g}$ i.p.). For direct toluidine blue staining, skin samples were torn down laterally without muscle tissue using $12 \mathrm{~mm}$ skin biopsy punches. The samples were stained with $1 \%$ toluidine blue without fixation. The lateral distribution of mast cells was examined by stereoscope (SMZ1000, Nikon, Japan). In the conventional toluidine blue staining experiment, skin samples were collected with muscle tissue using 5 $\mathrm{mm}$ skin biopsy punches. Sequential paraffin slices of $5 \mu \mathrm{m}$ thickness were made after $48 \mathrm{~h}$ of fixation at $4^{\circ} \mathrm{C}$ in fixing solution ( $10 \%$ formalin). The sections were longitudinal to the skin and the muscle tissue. The sample was stained with $0.5 \%$ toluidine blue on slides and examined using an inverted microscope (Ti-s, Nikon, Japan).

\section{Immunofluorescence staining}

The immunohistochemical staining method was performed in ST36 point skin tissues as described in a previous study [18]. The anaesthetized rats were immediately subjected to whole body perfusion through the heart with $100 \mathrm{~mL}$ of $0.9 \%$ saline, followed by $300 \mathrm{~mL}$ of $4 \%$ paraformaldehyde in $0.1 \mathrm{M}$ PBS ( $\mathrm{pH}=7.4)$. After perfusion, the skin tissue in the area of ST36 was dissected out from the right leg using $6 \mathrm{~mm}$ skin biopsy punches and stored in $25 \%$ 
sucrose solution at $4^{\circ} \mathrm{C}$. Skin sections from ST36 were cut at a thickness of $20 \mu \mathrm{m}$ on a cryostat (CM1900, Leica, Germany). The sequentially mounted slides were blocked with QuickBlock ${ }^{\mathrm{TM}}$ blocking buffer (Beyotime Biotech, Shanghai, China) and prepared for their respective types of fluorescence immunohistochemical staining. Primary antibodies, including mouse monoclonal anti-mast cell tryptase antibody (ab2378, 1:1000, Abcam) and rabbit polyclonal anti-beta III tubulin antibody (ab52623, 1:200, Abcam), were used in this study. Goat anti-mouse Alexa Fluor 594 secondary antibody (8890, 1:500, CST) and goat anti-rabbit Alexa Fluor 488 secondary antibody (150077, 1:500; Abcam) were used to visualize the corresponding primary antibodies. Additionally, DyLight $^{\mathrm{TM}} 488$ Phalloidin (12935, 1:500, CST) and 4',6-diamidino-2-phenylindole 2 hci (DAPI) (D1306, 1:40000, Molecular Probes, USA) were applied for counterstaining. The tissue samples were examined under a confocal laser scanning microscope (SP8, Leica, Germany).

Immunocytochemical staining was carried out on cells that adhered on the substrate. All reagents for fixation, washing, and blocking as well as dilution buffer were purchased from Beyotime (Beyotime Biotechnology, Shanghai, China). RBL-2H3 cells were grown on normal or PDMS substrate for 18 hours, rinsed in PBS ( $\mathrm{pH}=7.4)$ two times, fixed for 30 minutes at room temperature and exposed in $0.2 \%$ Triton X-100 in PBS for $4 \mathrm{~min}$. Then, the cells were blocked for 30 minutes at room temperature. The cells were incubated in DyLight ${ }^{\mathrm{TM}} 488$ Phalloidin and DAPI for 30 minutes at room temperature in the dark. The cell samples were examined under an inverted fluorescence microscope (Ti-e, Nikon, Japan).

\section{PDMS substrate}

PDMS is a widely used bio-compatible substrate for cell behavior studies [19, 20]. The Sylgard 184 PDMS (DowCorning, Midland, MI, USA) was prepared through mixing the curing agent and base in a specified mass ratio, and it was degassed in a vacuum chamber for 40 minutes at room temperature to remove the air bubbles from the mixtures. The mixtures were then poured into dishes to produce approximately $1 \mathrm{~mm}$ thick thin films. All samples were cured at $80^{\circ} \mathrm{C}$ for 6 hours in an oven to form the PDMS substrates. Then, these substrates were modified in terms of their hydrophilicity and sterilized under UV radiation for cell culture. Different mass ratios of curing agent and base get different stiffness substrate. The nanoindentation tests were performed in a Hysitron Ti-950 nanoindenter system equipped with a Berkovich fluid cell probe (TI-950, Hysitron, Inc., Minneapolis, MN, USA). The probe was engaged on the sample surface in $1 \%$ BSA (in PBS) solution with 0.5 micron Newton $(\mu \mathrm{N})$ force and withdraw $150 \mathrm{~nm}$ before each indentation test. During the test, the probe was loaded up to maximum force in 5 seconds, then held for 2 seconds, and unloaded to zero force in 5 seconds. The depth and force as a function of time is recorded simultaneously and the data was used to derive the Hardness and reduced Modulus of the materials using Oliver-Pharr model. The maximum forces are selected to reach the same maximum depth which is about 10 micron meter $(\mu \mathrm{m})$ for all tests.

There are some methods to determine the stiffness gradients or stiffness variations of the PDMS substrates, such as by UV irradiation through a printed mask [21]. In this study, we made the substrate with stiffness variations by pouring two different ratios of PDMS liquid into the opposite sides of culture dishes. Keeping the dish horizontal, we completed the rest of the steps according to the previously described general steps. We termed this method the free flow confluence (FFC) method. The details can be found in the supplementary material (Supplement. 1, Fig. S1). Black carbon powder was mixed into one side of the PDMS to illustrate the boundary of different ratios of PDMS only for convenient observation. PDMS substrates for culturing cells was transparent and without black carbon. PDMS substrate with stiffness variations by free flow confluence (FFC) method was detected by Hysitron TI-950 nanomechanical instrument.

\section{AO/PI method to count cell concentration and viability}

The acridine orange $(\mathrm{AO})$ and propidium iodide (PI) dual staining method has been routinely used to measure the number and viability of nucleated cells [22, 23]. A cell counter (Counter Auto 2000, Nexcelom Bioscience, Lawrence, MA, USA) was used to detect fluorescence and count the cells. First, the culture dish was gently shaken to collect the supernatant. The substrate was rinsed using PBS $(\mathrm{pH}=7.4)$ to mix the supernatant. Second, the supernatant was mixed. In total, $20 \mu \mathrm{L}$ was removed to be mixed with $\mathrm{AO} / \mathrm{PI}$ dye liquid at a ratio of 1:1 to count on a counter. All nonadherent cell concentrations and viability were determined. Immediately, we added $1 \mathrm{~mL}$ PBS $(\mathrm{pH}=7.4)$ to the dish from which the supernatant was removed. Cells were scraped on the substrate with a cell scrape, taken out and rinsed in the same dish with $1 \mathrm{~mL}$ PBS before being mixed and counted on a counter as mentioned above. Incorporation of annexin $\mathrm{V}$ and Brdu was also recommended method to determine the survival and proliferation of the cells affected by PDMS substrates. 


\section{Acupuncture stimulation}

The ST36 is located at the anterior tibia muscle near the knees according to previous studies [5,24]. It is a popular acupoint for studies in animal experiments as well as for clinical treatment. Sterilized stainless steel acupuncture needles (0.25 $\mathrm{mm}$ in diameter, 1 inch in length, Suzhou Kangnian Medical Devices, Jiangsu, China) were inserted into the ST36 at the right hind leg, located $5 \mathrm{~mm}$ lateral and distal to the anterior tubercle of the tibia. For the lifting-twisting acupuncture group, the perpendicular needling depth was approximately $5 \mathrm{~mm}$, and we alternately applied lift-thrusting and twisting manipulation for 30 seconds with 30 second intervals. The total acupuncture process was performed for 30 min. For the retention acupuncture group, the needle was inserted into the tissue as the lifting-twisting acupuncture group, but without motion. For the control group, there was no acupuncture treatment; the other treatments were the same as the above two groups.

\section{Statistical analysis}

The data are presented as the standard error of the mean (SEM). The statistical analysis was performed using 1-way analysis of variance (one-way ANOVA) with the Student-Newman-Keuls (S-N-K) post hoc test. In all of the analyses, the differences were considered statistically significant at $p<0.05$. All statistical analyses were performed using SPSS 20.0 (International Business Machines, Armonk, NY, USA).

\section{Results}

\section{Mast cells are distributed in a spatially nonuniform manner}

Mature mast cells are recognized in the skin histochemically by the ability of their intracellular granules to bind to toluidine blue and other cationic dyes [25]. Previous research has examined mast cells using the toluidine blue staining method on paraffin or frozen sections along the longitudinal direction of the skin [26, 27]. To determine the spatial distribution of mast cells in the skin, we examined mast cells along a longitudinal and lateral directions. Rat skin samples were taken from the right leg around the ST36 using skin biopsy punches (Fig. 1A) and spread naturally by a homemade skin gripper after direct toluidine blue staining (Fig. 1B). The lateral distribution of mast cells was examined by stereoscope (Fig. 1C). From the enlarged figure (Fig. 1D), the lateral level of distribution of mast cells is determined, and the result shows that mast cells are distributed widely but unequally in a lateral direction to the skin. The longitudinal distribution of mast cells was examined by microscope on paraffin sections (Fig. 1E). In the longitudinal direction, mast cells are also distributed widely and unequally. The enlarged part (Fig. 1F) shows mast cells indicated by yellow arrows, dyed blue and purple, with different shapes.
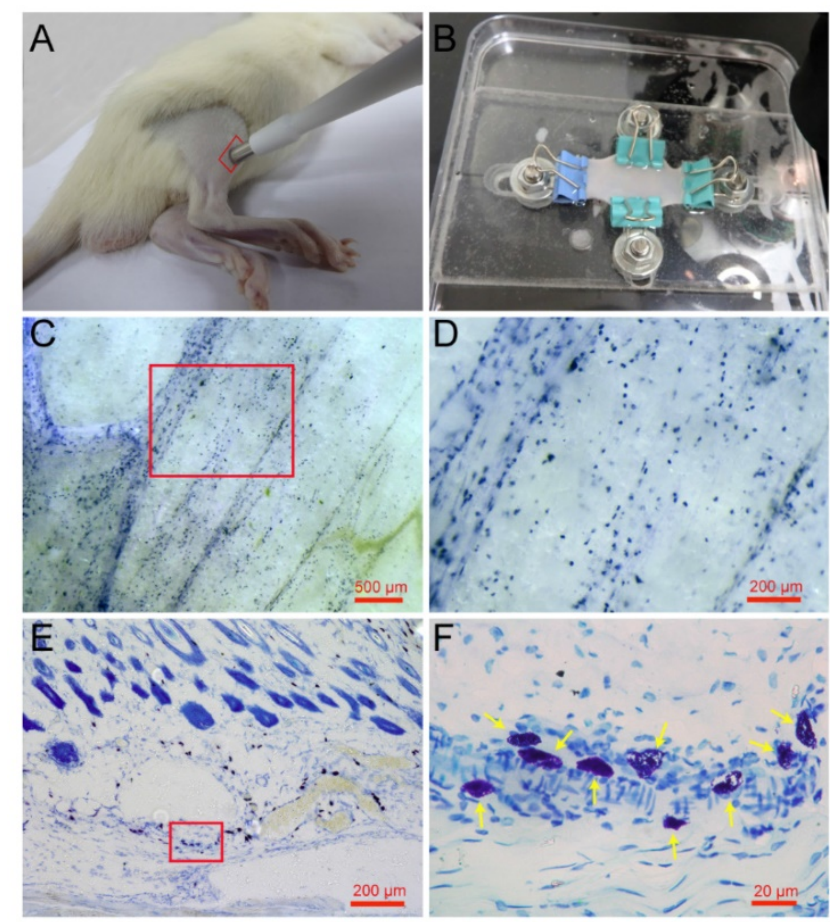

Figure 1. Spatial distribution of mast cells in rat skin. (A) Skin samples were taken from a rat leg around the ST36 using a skin biopsy punch. (B) Direct toluidine blue stained skin sample was spread naturally by a homemade skin gripper for stereoscopic examination. (C) The lateral distribution of mast cells was examined by stereoscope. (D) Enlargement of the red frame area in (C). (E) The longitudinal distribution of mast cells examined by microscope. (F) Enlargement of the red frame area in $(\mathrm{E})$, with mast cells indicated by yellow arrows. The scale bar is shown in the lower right corner of each examined figure.

\section{Mast cells are distributed along hair follicles, vessels, nerve fibers, adipose tissue, and muscle tissue in the rat skin}

Hair follicles, vessels, nerve fibers, and adipose tissue are the most important and functional tissues of the skin. Beneath the skin, muscle tissue is always closely linked. We examined the location relationship between mast cells and these functional tissues separately using immunofluorescence staining on frozen sections and toluidine blue staining on paraffin sections. Correlation of mast cells and hair follicles (Fig. 2A-A3), vessels (Fig. 2B-B3), and nerve fibers (Fig. 2C-C3) by immunofluorescence staining shows that mast cells are located at the boundary of these tissues. Mast cells are also located along adipose tissue (Fig. 3A-B) and muscle tissue (Fig. 3C-D), as shown by toluidine blue staining of the samples. 
Specific tissues were circled with a rectangle area $(400 \mu \mathrm{m} \times 250 \mu \mathrm{m})$ in staining samples. The number of mast cells adjacent to specific tissues $(\mathrm{d}<20 \mu \mathrm{m})$ or distant to specific tissues $(\mathrm{d}>20 \mu \mathrm{m})$ were counted out ( $d$ represents the vertical distance from one cell to specific tissue). In order to illustrate this procedure, supplementary material was added (Supplement. 2, Fig. S2) for detail explanation. Statistical results of the number of adjacent and distant cells around five kinds of specific tissues were present (Fig. 3E).
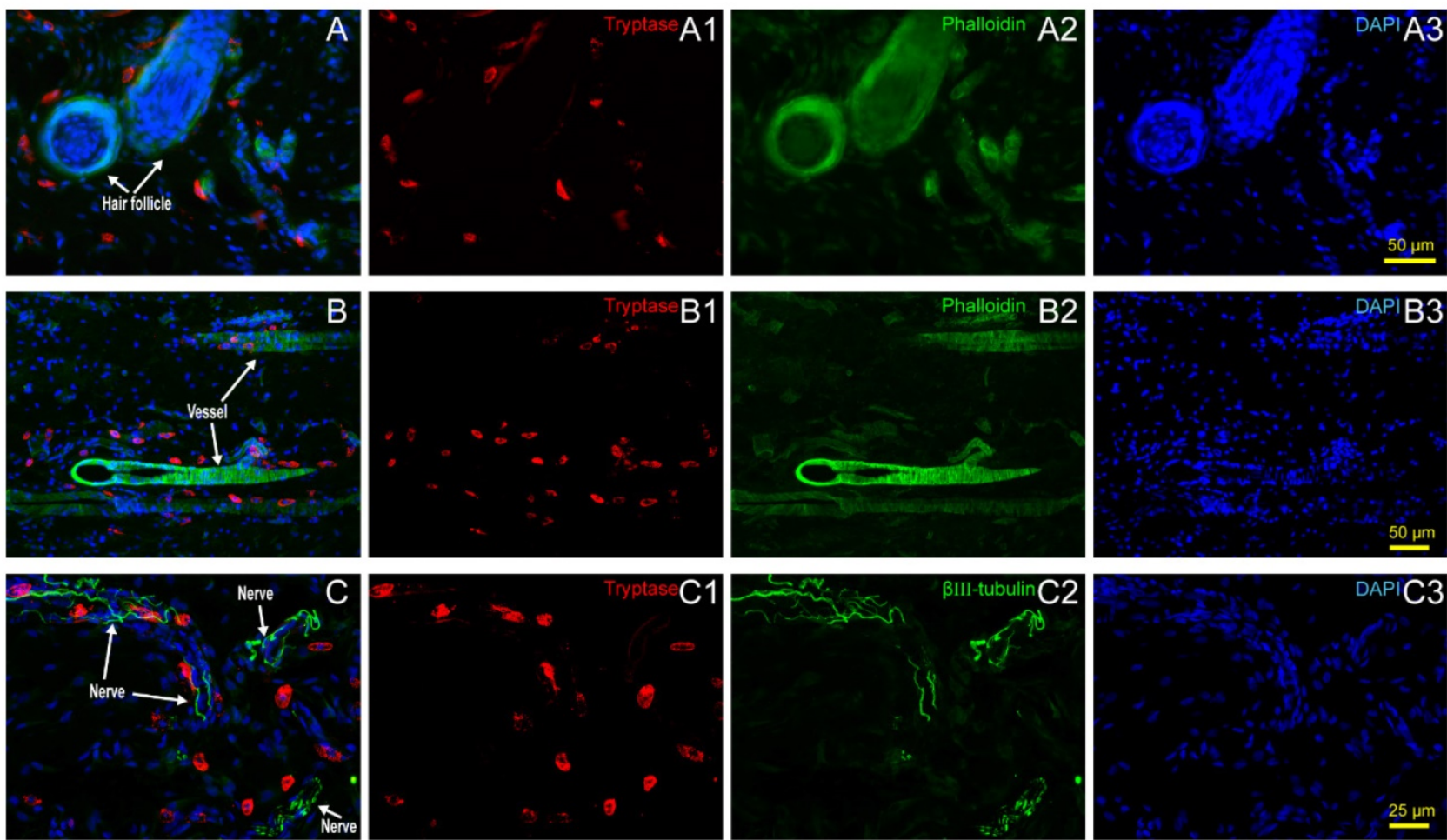

Figure 2. Mast cell distribution along hair follicles, vessels and nerve fibers. (A) Correlation of mast cells and hair follicles in skin examined with immunofluorescence histochemical staining with (A1) tryptase (red), (A2) phalloidin (green), and (A3) DAPI (blue). (B) Correlation of mast cells and vessels in skin samples examined with immunofluorescence histochemical staining with (B1) tryptase (red), (B2) phalloidin (green), and (B3) DAPI (blue). Hair follicles and vessels are distinguished by their location and morphological characteristics. (C) Correlation of mast cells and hair follicles in skin examined with immunofluorescence histochemical staining with (C1) tryptase (red), (C2) $\beta$-III tubulin (green), and (C3) DAPI (blue). Scale bars are shown in the DAPI-stained figure in each group.
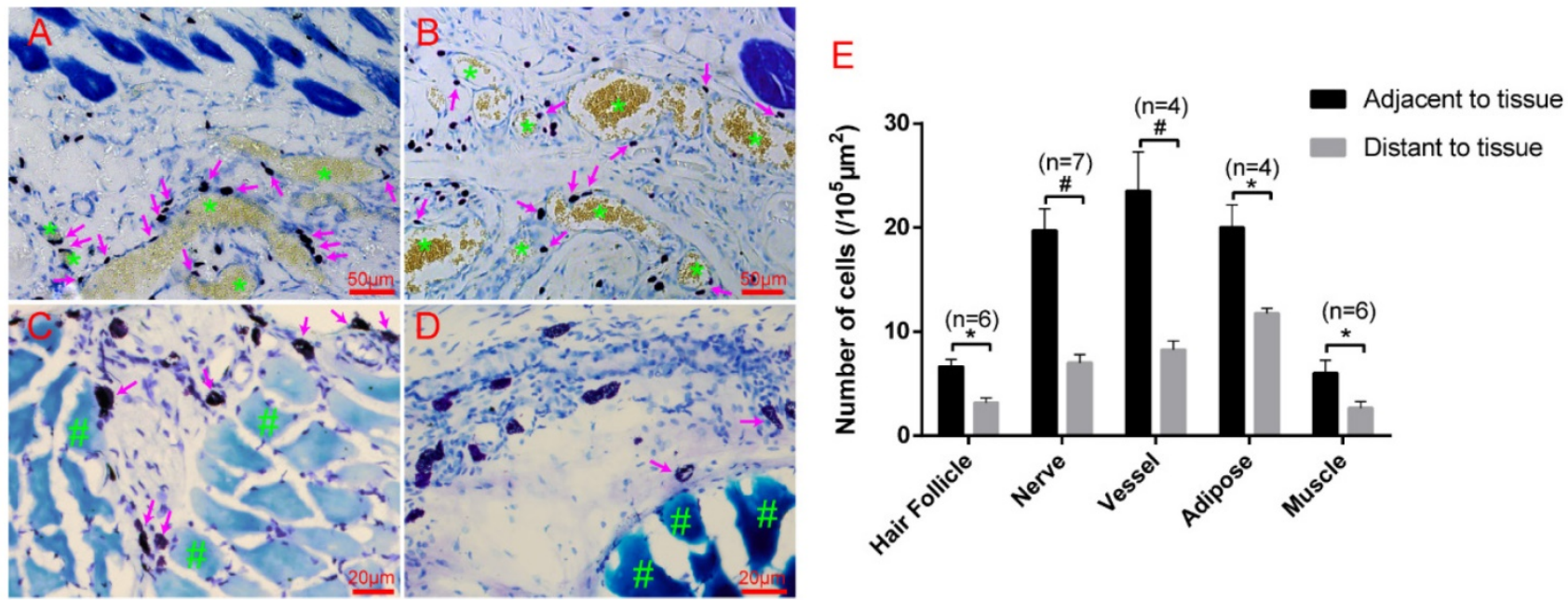

Figure 3. Mast cells are distributed along adipose and muscle tissues. (A-B) Mast cells are distributed along adipose tissue. Adipose tissue is dyed yellow with gathered particles and marked by green asterisks '*' in the figures. (C-D) Mast cells are distributed along the muscle tissue. The muscle tissue is dyed blue or dark blue and marked by green pound signs '\#' in the figures. Mast cells around the tissue boundaries are marked with magenta arrows, and the scale bar is shown in the lower right-hand corner of each examined figure. (E) Statistical results of the number of adjacent and distant cells around five kinds of specific tissues. Intra-group comparison difference in each group $* p<0.05, \# p<0.01$. 
A

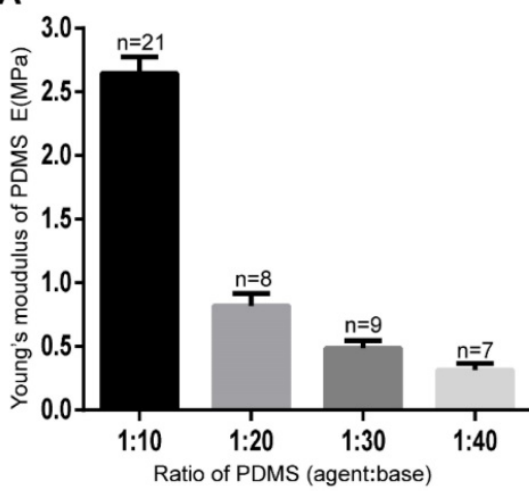

B

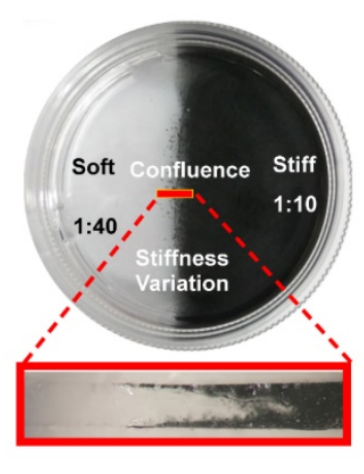

C1

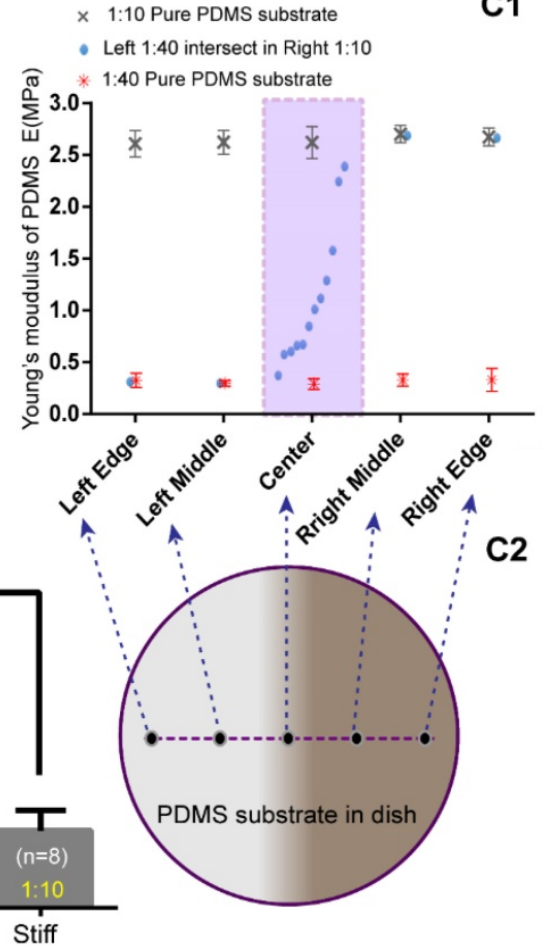

D1

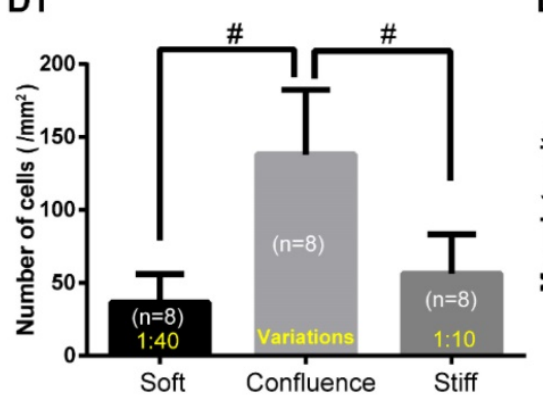

02

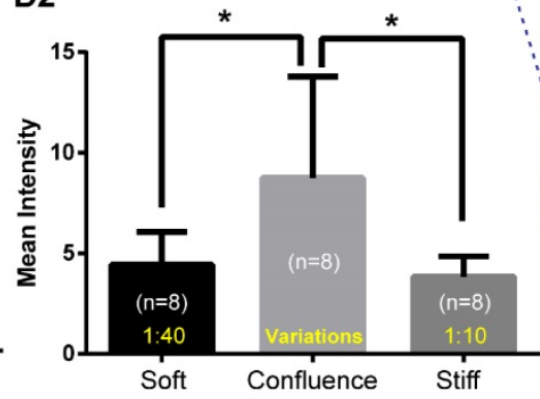

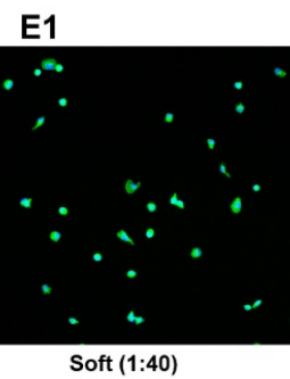

E2

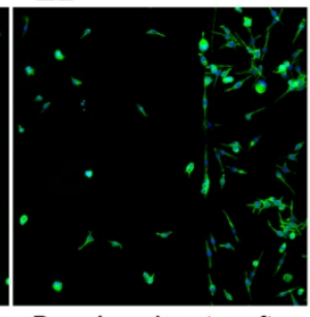

Boundary close to soft
E3

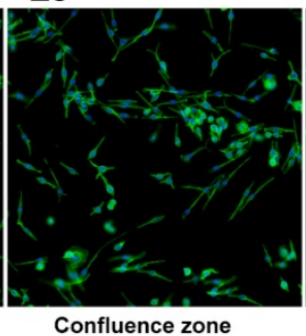

E4

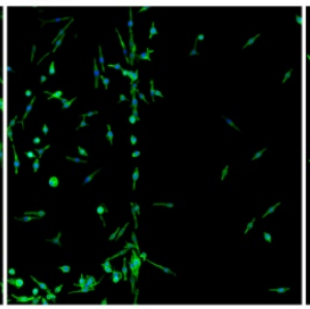

Boundary close to stiff
E5

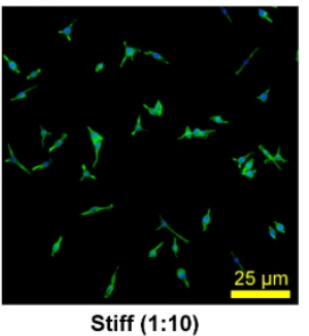

Figure 4. PDMS substrate stiffness properties and the distribution of RBL-2H3 cells on the PDMS substrate. (A) The Young's modulus of different ratios of PDMS was detected by Hysitron TI-950 nanomechanical instrument. The ratios of PDMS (agent:base) were 1:10, 1:20, 1:30, and 1:40. (B) Cell culture dish (diameter $=35 \mathrm{~mm}$ ) coated with PDMS substrate with stiffness variations using the FFC method. The sectional drawing of the area with stiffness variation of the soft PDMS (agent:base $=1: 40$ ) and stiff PDMS (agent:base=1:10) which was marked with black carbon powder. (C1-C2) Young's modulus of substrate with stiffness variations was detected point-by-point along the diameter of the dish. Pure 1:10 and pure 1:40 PDMS substrates were also detected for comparison. (D1) The adherent cell number of every $1 \mathrm{~mm}^{2}$ on substrates that are soft $(1: 40)$, stiff $(1: 10)$ or with stiffness variations. (D2) The adherent cells correspond to fluorescence intensities on the substrates that are soft (1:40) or stiff (1:10) or that have a stiffness variation band. *p<0.05 versus boundary group, \#p<0.01 versus boundary group. Values are given as the means \pm SE. (E1) Cells on the softer PDMS substrate (agent:base=1:40). (E2) Cells on the boundary of the softer side of the PDMS substrate with stiffness variation. (E3) Cells on the PDMS substrate with stiffness variation. (E4) Cells on the boundary of the stiffer side of the PDMS substrate with stiffness variation. (E5) Cells on the stiffer PDMS substrate (agent:base=1:10). All cells are RBL-2H3 mast cells, in which the cytoskeleton is dyed green by phalloidin, and the nuclei are dyed blue by DAPI. The scale bar is $25 \mu \mathrm{m}$.

\section{RBL-2H3 cells are distributed along the areas with stiffness variations of the PDMS substrate.}

To exam mast cell distribution properties on the substrate with stiffness variations in vitro, we made substrates with stiffness variations using PDMS. The Young's modulus of PDMS with different mass ratios of PDMS (agent:base) was tested using Hysitron TI-950 nanomechanical instrument. We observed a remarkable change from 1:10 to 1:40 (Fig. 4A). Cell culture dishes with the stiffness variations of PDMS substrates were made with two different mass ratios of agent:base, the softer of which is 1:40, and the stiffer is 1:10 (Fig. 4B). PDMS substrates with stiffness variations were made by free flow confluence (FFC) method. Black carbon powder which mixed into one side of the PDMS illustrated that there is a transitional region between softer and stiffer PDMS. It should be noted that the black carbon powder is only used to illustrate the boundary properties of different ratios of PDMS confluence and the RBL-2H3 cells were cultured on PDMS without black carbon powder. Young's modulus of substrates with stiffness variations were detected by Hysitron TI-950 nanomechanical instrument. Comparing with pure 
1:10 and pure 1:40 substrates, PDMS with stiffness variations presented an ideal range of hardness gradient (Fig 4C1-D2). RBL-2H3 cells were cultured in dishes with PDMS substrate, and the initial cell concentration was $5 \times 10^{5} / \mathrm{mL}$ in every dish. After culturing 18 hours, the adherent cells were dyed green by phalloidin to examine the cell distribution and the number of cells and mean fluorescence intensity in the softer areas, variation areas and stiffer areas were counted (Fig. 4D1-D2). Cells' number and fluorescence intensity in the confluent area were higher than those in the softer or stiffer PDMS substrate. RBL-2H3 cells in the stiffness variation area were presented in supplementary material (Supplement. 3, Fig. S3). Five typical areas were identified: the softer side, the boundary with the softer side, the confluent area, the boundary with the stiffer side, and the stiffer side with the same latitude in the same dish (Fig. 4E1-E5).

Based on the above results, we can infer that there are two possible reasons for mast cell recruitment on the areas with stiffness variations. One possibility is that the areas with stiffness variations is more suitable for RBL-2H3 cell growth and reproduction. The other possibility is that RBL-2H3 cells tend to migrate in the direction of the areas with stiffness variations.

To determine if the different stiffness or stiffness variations of PDMS substrates affects the growth and viability of mast cells, we detected the viability and concentration of RBL-2H3 cells in varying substrate dishes using the AO/PI method [23]. The initial cell concentration was $5 \times 10^{5} / \mathrm{mL}$, and the culture time was 18 hours. Different fields of suspended cells and adherent cells were captured by the cell counter (Fig. 5B1-B4, C1-B4), and the cell counter auto counted the live and dead cells depending on the fluorescence color (Fig. 5B5, C5). There are 6 groups of characteristic cell culture substrates. The concentration and viability of the suspended or not attached cells increased with the decrease in rigidity (Fig. 5A1). The number of attached cells decreased with the decrease in rigidity (Fig. 5A2). Combined with the total cell concentration and viability (Fig. 5A3), we can identify that the growth ability of RBL-2H3 cells on the areas with stiffness variations is between the stiffer and softer substrates. This result verifies that the reasons for mast cell recruitment on the areas with stiffness variations is that mast cells tend to migrate in the direction of the areas with stiffness variations.
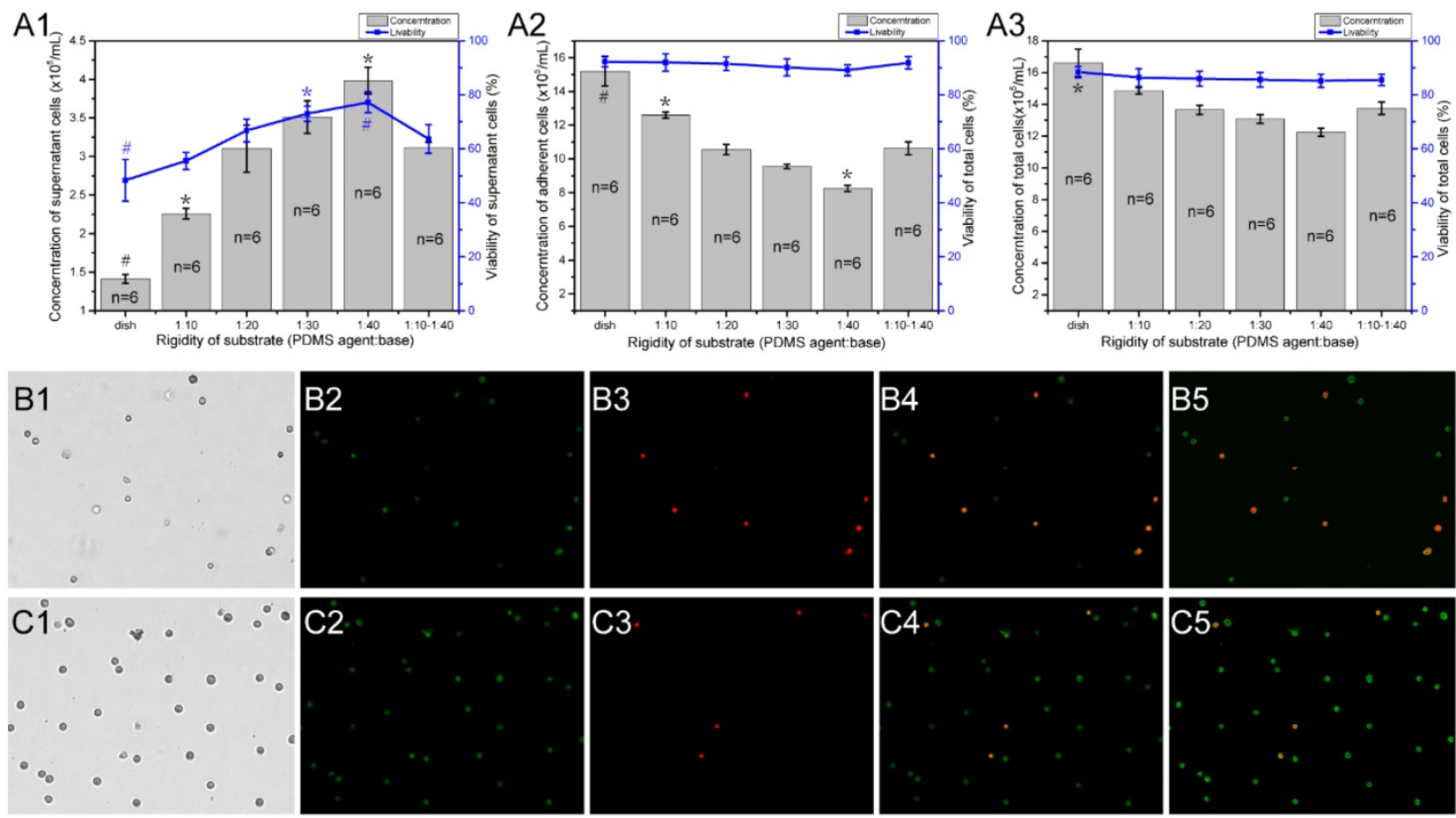

Figure 5. The concentration and viability of RBL-2H3 cells on different rigidity regions of PDMS substrates. Cell culture dishes were used with different substrates as follows: normal cell culture dishes without coating PDMS (dish), dishes coated with a single stiffness PDMS substrate with a specified ratio of agent:base $(1: 10,1: 20,1: 30$, and 1:40), and dishes coated with a stiffness gradient PDMS substrate with two intersecting sides: the stiffer side is 1:10, and the softer side is 1:40 (1:10-1:40). ( $\mathrm{Al})$ The concentration and viability of cells that did not adhere to the substrate. (A2) The concentration and viability of cells that adhered to the substrate. (A3) The concentration and viability of whole cells in the culture dish. The gray columns represent the number of cells. The blue line represents the survival rate of the cells. ${ }^{*} p<0.05$ versus 1:10-1:40 group, $\# p<0.01$ versus 1:10-1:40 group. Values are given as the means \pm SE. $(B 1-B 5)$ and $(C 1-C 5)$ The $A O / P I$ method is used to determine the concentration and viability of cells that are not attached to and attached to the substrate, respectively. 


\section{Discussion and conclusions}

The ability of cells to follow gradients of extracellular matrix stiffness was termed durotaxis by Lo et al and was established as a single cell phenomenon [28]. Sunyer et al recently found that durotaxis also directs the motion of cell collectives, and it emerged from long-range intercellular force transmission [29]. Some experimental studies have revealed parts of molecular biological mechanisms [30-32]. Additionally, numerical simulations have tried to establish mathematical models to explain this phenomenon [33-36]. At present, its specific mechanism is still unresolved.

In the present study, the following results were achieved. (1) Through the examination of tissue sections of rat skin, we found that mast cells are distributed widely but unequally and usually localize to the areas with stiffness variations in tissues, such as hair follicles, vessels, nerves, adipose tissue, and muscle tissue in vivo. (2) Through culturing RBL-2H3 cells on PDMS substrates and testing their number and viability, it was found that mast cells are distributed along the stiffness gradient of PDMS substrates in vitro. Combined with the latest research progress about the origin and location of mast cells, a hypothesis about the distribution of mast cells in known or unknown tissues was formed: (1) If a tissue contains mast cells, it should contain permeable capillaries and a suitable bio-factor environment. (2) If there are mast cells in the tissue, their distribution follows durotaxis properties. Of course, there are other factors, such as histamine [37], LTC4 [38], ATP [39], TNF-a and IL-4 [40], that act in mast cell chemotaxis. To our knowledge, this study is one of the few studies [41] to report mast cell migration related to durotaxis in vivo and in vitro and to suggest the existence and location of mast cells in various tissues.

To know how mast cells in specific tissues are distributed is important for an understanding of the functional mechanisms of mast cells involved in bio-processes. We introduce another two areas where the existence and distribution of mast cells are critical besides the skin: the eyes and the blood brain barrier. In humans, mast cells are abundant in the anterior and posterior uvea but absent in the retina [42]. In hatching and adult birds, mast cells are absent in the cornea and retina [43]. In dogs, the mast cell marker chymase was not detected in the cornea [44]. The cornea is one kind of tissue without capillaries, and the retina has no permeable capillaries within it [45]. These phenomena may verify our hypothesis that tissues containing mast cells should have permeable capillaries and suitable bio-factor environments. The blood-brain barrier is a specialized structure formed by the blood vessels of the central nervous system, and it tightly restricts the flow of blood-borne ions, molecules, and cells from entering the neural tissue [46]. However, mast cells are located at the perivascular region close to nerve endings, and they can respond to and release mediators of both the nervous and immune system while regulating blood-brain barrier permeability [47, 48]. In this structure, the stiffness gradient provides a link between glial tissue and endothelial tissue, which form the blood brain barrier. Mast cells are also distributed along the gut immune barrier [46]. These phenomena may verify our hypothesis that the distribution of mast cells follows durotaxis properties.

Combined with previous studies about the origin of mast cells $[49,50]$ and the results we report, we present a diagrammatic sketch to show the origin and strategic distribution of mast cells in the skin (Fig. 6). Mast cell progenitors emerge from the bone marrow and remain immature while circulating in blood vessels. Immature mast cells can permeate capillaries and settle in specified tissues. The microenvironment surrounding the mast cells determines their mature phenotype. This process is shown in a supplementary video (Video. S1).

Mast cells are provisioned with multiple types of receptors as well as mediators, which provide a reasonable explanation for the multifunctional role of these cells [9]. According to our finding that mast cells are distributed along vessels, nerves, hair follicles, adipose tissues, and muscle tissues, the meaning and function of these distribution structures should be discussed.

The vessels include blood vessels and lymphatic vessels. Blood vessels are blood flow pathways in the circulatory system. The phenomenon in which mast cells are distributed along vessels was described by Paul Ehrlich when they were first identified [51]. Currently, we know that mast cells contain heparin [52] and histamine [7], which can change the permeability of the vessels and acquire $\operatorname{IgE}$ by extending cell processes across the vessel wall [53]. This reveals a two-way interaction strategy of mast cells and the vasculum. Mast cell-vessel interactions are detailed in our supplementary video (Video. S2). Mast cells have been found around the peripheral and central systems [54]. Mast cells release inflammatory mediators, including histamine, 5-HT, or cytokines, that directly activate neurons to mediate physiopathology in the peripheral and central nervous systems. Neurons also release neurotransmitters and neuropeptides that directly act on mast cells to modulate their function [55]. Neuro-mast cell interactions studies founded the cross-talk between the immune system and nervous system and could lead to a better understanding of therapy methods 


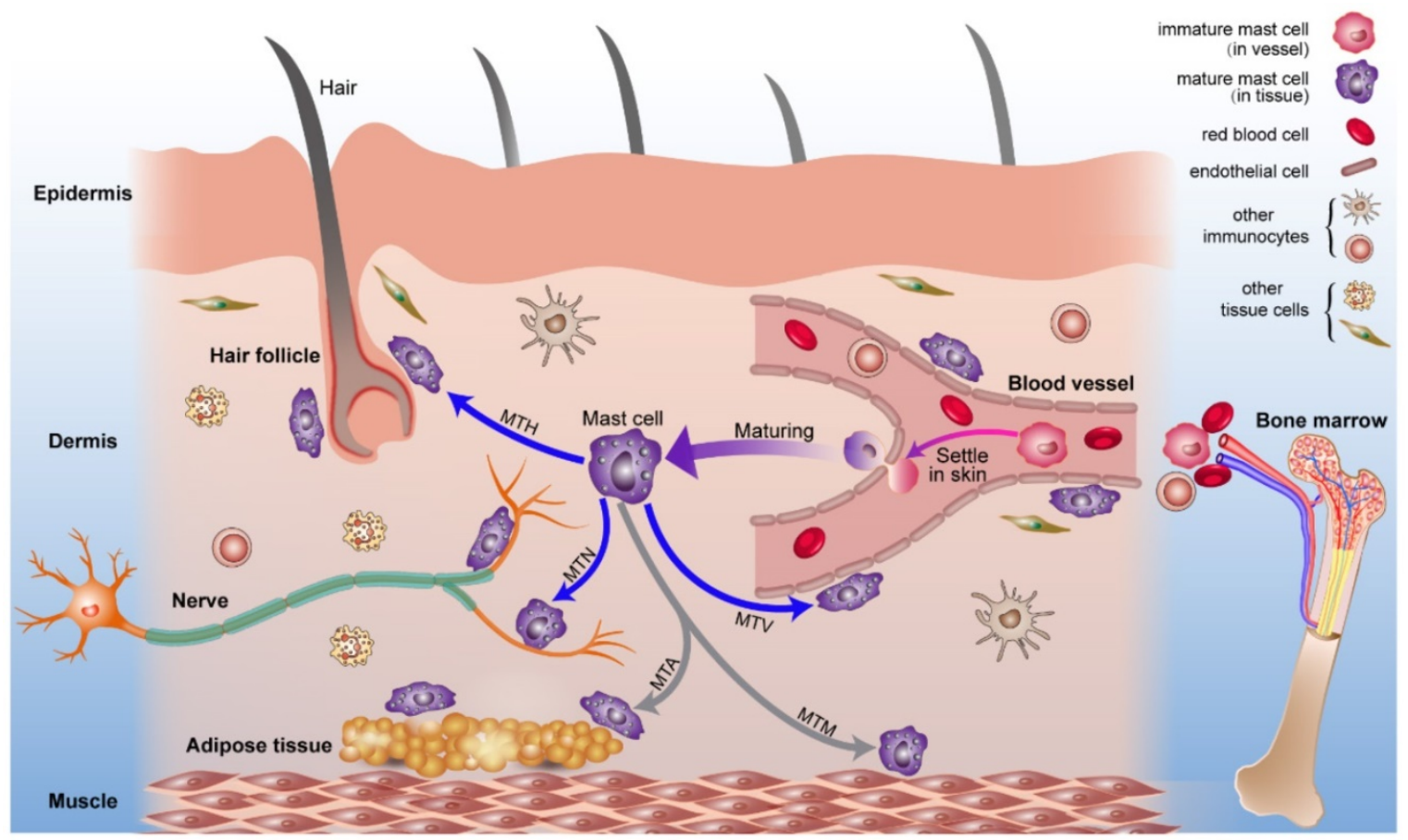

Figure 6. The origin, circulation, maturation, migration and strategic location of mast cells in normal skin. Mast cell progenitors originate from bone marrow and circulate in blood vessels. Under certain conditions, immature mast cells can leak out of capillaries and settle into the skin. Mast cells migrate to vessels, nerves, hair follicles, muscle tissue, and adipose tissue and are distributed within them. Mast cells can modulate the behavior of these neighboring tissues rapidly through the release of mediators under this distribution pattern. Abbreviations: MTH, migration to hair follicles; MTN, migration to nerves; MTA, migration to adipose tissue; MTM, migration to muscle tissue; MTV, migration to vessels.

such as acupuncture for the treatment of some diseases [56]. Mast cell-nerve interactions are detailed in our supplementary video (Video. S3). The interaction between mast cells and adipose tissue [57], muscle tissue [58] and hair follicles [59, 60] also provides information on bioprocesses.

In pathological conditions and tissue repair duration, some factors always cause local tissue fibrosis $[61,62]$. There is a positive trend toward the correlation of mast cell numbers with fibrosis [63-66]. Based on the hypothesis above, this phenomenon could be explained by the local stiffness gradient, which should be an important mechanical factor for the recruitment of mast cells. Traditional manual acupuncture might be a way to modify the stiffness of local tissues through the lifting and twisting of needles. Through applying different motions of acupuncture, we found that mechanical stimulation induced the accumulation and degranulation of mast cells in the stress-changing region (Supplement. 4, Fig. S4). Diverse substances released from mast cells could initiate local biochemical process and nerve signals to central for contributing to acupuncture effects. This processes provided a clue to reveal the mechanism of acupuncture. However, our results in manual acupuncture mixed with a variety of factors. The migration of mast cells under changing stiffness should be studied further in vitro.
In summary, this study examined the distribution and location of mast cells around several main tissues and verified their unequal spatial distribution in the rat skin. In the in vitro experiment, we verified that RBL-2H3 cells tend to be distributed along the areas with stiffness variations. Based on the experimental results and the origin of the mast cells, we formed a hypothesis about the distribution and location of mast cells in the identified or unidentified tissues. That is mast cells distribute in the areas with stiffness variations and locate along the stiffness gradient formed by tissue and extracellular matrix. By applying different motions of acupuncture in the rat skin, we determined that the changing mechanical stimulation induced the accumulation and degranulation of mast cells. This finding suggests that acupuncture may produce the proper treatment effects by modifying the local stiffness (Supplement. 4, Fig. S5). Studies on the migration, distribution, matrix circumstance and mechanical response of mast cells may be important for understanding the role of mast cells in physiological and pathological processes. Furthermore, we can modify the stiffness of local tissues to determine the specified treatment for some diseases.

\section{Acknowledgments}

This work was supported by the National Natural Science Foundation of China (81473750, 
81574053, 81590953, and 11372191), Shanghai Key Laboratory of Acupuncture Mechanism and Acupoint Function (14DZ2260500), and the Science and Technology Commission of Shanghai Municipality (15441903800 and 18401970100).

\section{Supplementary Material}

Supplementary methods and figures.

http://www.ijbs.com/v14p1142s1.pdf

Supplementary video $\mathrm{S1}$.

http://www.ijbs.com/v14p1142s2.mp4

Supplementary video $\mathrm{S} 2$.

http://www.ijbs.com/v14p1142s3.mp4

Supplementary video $\mathrm{S3}$.

http://www.ijbs.com/v14p1142s4.mp4

\section{Competing Interests}

The authors have declared that no competing interest exists.

\section{References}

1. Stelekati E, Orinska Z, Bulfone-Paus S. Mast cells in allergy: Innate instructors of adaptive responses. Immunobiology. 2007; 212:505-19.

2. Galli SJ, Grimbaldeston M, Tsai M. Immunomodulatory mast cells: negative, as well as positive, regulators of immunity. Nat Rev Immunol. 2008; 8:414-78.

3. Norrby K. Mast cells and angiogenesis. Apmis. 2002; 110:355-71.

4. Tellechea A, Lea EC, Kafanas A, Auster ME, Kuchibhotla S, Ostrovsky Y, et al. Mast Cells Regulate Wound Healing in Diabetes. Diabetes. 2016; 65:2006-19.

5. Zhang D, Ding G, Shen X, Yao W, Zhang Z, Zhang Y, et al. Role of Mast Cells in Acupuncture Effect: A Pilot Study. EXPLORE: The Journal of Science and Healing. 2008; 4:170-7.

6. Zhu H, Wang X, Huang M, Jing Y, Zhang D, Ding G. Mast cell activation in the acupoint is important for the electroacupuncture effect against pituitrin-induced bradycardia in rabbits. Sci Rep-Uk. 2017; 7.

7. Metcalfe D D BDMY. Mast Cells. Physiol Rev. 1997; 77:1033-79.

8. Crivellato E, Beltrami CA, Mallardi F, Ribatti D. Paul Ehrlich's doctoral thesis: A milestone in the study of mast cells. Brit J Haematol. 2003; 123:19-21.

9. Beaven MA. Our perception of the mast cell from Paul Ehrlich to now. Eur J Immunol. 2009; 39:11-25

10. Blank U, Falcone FH, Nilsson G. The history of mast cell and basophil research - some lessons learnt from the last century. Allergy. 2013; 68:1093-101.

11. Yang W. Investigation of the Lower Resistance Meridian: Speculation on the Pathophysiological Functions of Acupuncture Meridians. Evid-Based Compl Alt. 2014; 2014:1-7.

12. Stokes AJ, Shimoda LM, Koblan-Huberson M, Adra CN, Turner H. A TRPV2-PKA signaling module for transduction of physical stimuli in mast cells. J Exp Med. 2004; 200:137-47.

13. Hershkoviz R, Preciado-Patt L, Lider O, Fridkin M, Mekori YA. Mast cell adhesion to extracellular matrix: local effects of acute phase reactants. Int Arch Allergy Immunol. 1997; 113:295-6.

14. Levental I, Georges PC, Janmey PA. Soft biological materials and their impact on cell function. Soft Matter. 2007; 3:299-306.

15. Weber A, Knop J, Maurer M. Pattern analysis of human cutaneous mast cell populations by total body surface mapping. Br J Dermatol. 2003: 148:224-8.

16. Tamma R, Guidolin D, Annese T, Tortorella C, Ruggieri S, Rega S, et al. Spatial distribution of mast cells and macrophages around tumor glands in human breast ductal carcinoma. Exp Cell Res. 2017; 359:179-84.

17. Tikoo S, Barki N, Jain R, Zulkhernain NS, Buhner S, Schemann M, et al. Imaging of mast cells. Immunol Rev. 2018; 282:58-72.

18. Wu M, Xu D, Bai W, Cui J, Shu H, He W, et al. Local cutaneous nerve terminal and mast cell responses to manual acupuncture in acupoint LI4 area of the rats. J Chem Neuroanat. 2015; 68:14-21.

19. Fuard D, Tzvetkova-Chevolleau T, Decossas S, Tracqui P, Schiavone P. Optimization of poly-di-methyl-siloxane (PDMS) substrates for studying cellular adhesion and motility. Microelectron Eng. 2008; 85:1289-93.

20. Gutekunst SB, Grabosch C, Kovalev A, Gorb SN, Selhuber-Unkel C. Influence of the PDMS substrate stiffness on the adhesion of Acanthamoeba castellanii. Beilstein J Nanotechnol. 2014; 5:1393-8.

21. Raczkowska J, Prauzner-Bechcicki S. Precise positioning of cancerous cells on PDMS substrates with gradients of elasticity. Biomed Microdevices. 2016; $18: 90$
22. Wallen $C A$, Higashikubo $R$, Dethlefsen LA Comparison of two flow cytometric assays for cellular RNA--acridine orange and propidium iodide. Cytometry. 1982; 3:155-60.

23. Chan LL, Smith T, Kumph KA, Kuksin D, Kessel S, Dery O, et al. A high-throughput $\mathrm{AO} / \mathrm{PI}$-based cell concentration and viability detection method using the Celigo image cytometry. Cytotechnology. 2016; 68:2015-25.

24. Chang S, Tsai C, Lin J, Hsieh C, Lin R, Cheng J. Involvement of serotonin in the hypoglycemic response to $2 \mathrm{~Hz}$ electroacupuncture of zusanli acupoint (ST36) in rats. Neurosci Lett. 2005; 379:69-73.

25. Douaiher J, Succar J, Lancerotto L, Gurish MF, Orgill DP, Hamilton MJ, et al. Development of Mast Cells and Importance of Their Tryptase and Chymase Serine Proteases in Inflammation and Wound Healing. In: Alt FW, ed. Advances in Immunology. Elsevier Science \& Technology, 2014:211-252.

26. Huang M, Zhang D, Sa Z, Xie Y, Gu C, Ding G. In Adjuvant-Induced Arthritic Rats, Acupuncture Analgesic Effects Are Histamine Dependent: Potential Reasons for Acupoint Preference in Clinical Practice. Evid-Based Compl Alt. 2012; 2012:1-6.

27. Mingfu L, Xiaotong D, Xiaojing S, Jin J, Jinling Z, Ying H. Study on the Dynamic Compound Structure Composed of Mast Cells, Blood Vessels, and Nerves in Rat Acupoint. Evid-Based Compl Alt. 2013; 2013:1-4.

28. Lo CM, Wang HB, Dembo M, Wang YL. Cell movement is guided by the rigidity of the substrate. Biophys J. 2000; 79:144-52.

29. Sunyer R, Conte V, Escribano J, Elosegui-Artola A, Labernadie A, Valon L, et al. Collective cell durotaxis emerges from long-range intercellular force transmission. Science. 2016; 353:1157-61.

30. Kai F, Laklai H, Weaver VM. Force Matters: Biomechanical Regulation of Cell Invasion and Migration in Disease. Trends Cell Biol. 2016; 26:486-97.

31. Schaefer A, Hordijk PL. Cell-stiffness-induced mechanosignaling - a key driver of leukocyte transendothelial migration. J Cell Sci. 2015; 128:2221-30.

32. Plotnikov SV, Pasapera AM, Sabass B, Waterman CM. Force fluctuations within focal adhesions mediate ECM-rigidity sensing to guide directed cell migration. Cell. 2012; 151:1513-27.

33. Novikova EE, Raab M, Discher DE, Storm CK. Persistence-driven durotaxis:generic, directed motility in rigidity gradients. Phys Rev Lett. 2017; $118 \cdot 1$

34. Aubry D, Gupta M, Ladoux B, Allena R. Mechanical link between durotaxis, cell polarity and anisotropy during cell migration. Phys Biol. 2015; 12:26008.

35. Stefanoni F, Ventre M, Mollica F, Netti PA. A numerical model for durotaxis. J Theor Biol. 2011; 280:150-8.

36. Allena R, Scianna M, Preziosi L. A Cellular Potts Model of single cell migration in presence of durotaxis. Math Biosci. 2016; 275:57-70.

37. Malone DG, Irani AM, Schwartz LB, Barrett KE, Metcalfe DD. Mast cell numbers and histamine levels in synovial fluids from patients with diverse arthritides. Arthritis Rheum. 1986; 29:956-63.

38. Yao W, Huang H, Ding G. A dynamic model of calcium signaling in mast cells and LTC4 release induced by mechanical stimuli. Chinese Science Bulletin. 2014; 59:956-63.

39. Wang L, Sikora J, Hu L, Shen X, Grygorczyk R, Schwarz W. ATP Release from Mast Cells by Physical Stimulation: A Putative Early Step in Activation of Acupuncture Points. Evid-Based Compl Alt. 2013; 2013:1-7.

40. Mak M, Spill F, Kamm RD, Zaman MH. Single-Cell Migration in Complex Microenvironments: Mechanics and Signaling Dynamics. J Biomech Eng. 2016; 138:21004.

41. Fung PCW. Probing the mystery of Chinese medicine meridian channels with special emphasis on the connective tissue interstitial fluid system, mechanotransduction, cells durotaxis and mast cell degranulation. Chin Med-Uk. 2009; 4:10.

42. McLeod DS, Bhutto I, Edwards MM, Gedam M, Baldeosingh R, Lutty GA Mast Cell-Derived Tryptase in Geographic Atrophy. Invest Ophthalmol Vis Sci. 2017; 58:5887-96

43. Oliani SM, Girol AP, Smith RL. Gap junctions between mast cells and fibroblasts in the developing avian eye. Acta Anat (Basel). 1995; 154:267-71.

44. Shiota N, Saegusa Y, Nishimura K, Miyazaki M. Angiotensin II-generating system in dog and monkey ocular tissues. Clin Exp Pharmacol Physiol. 1997; 24:243-8.

45. Yu DY, Yu PK, Cringle SJ, Kang MH, Su EN. Functional and morphological characteristics of the retinal and choroidal vasculature. Prog Retin Eye Res. 2014; 40:53-93.

46. Daneman $R$, Rescigno M. The gut immune barrier and the blood-brain barrier: are they so different? Immunity. 2009; 31:722-35.

47. Theoharides TC, Zhang B. Neuro-inflammation, blood-brain barrier, seizures and autism. J Neuroinflammation. 2011; 8:168.

48. Ribatti D. The crucial role of mast cells in blood-brain barrier alterations. Exp Cell Res. 2015; 338:119-25.

49. Dahlin JS, Hallgren J. Mast cell progenitors: origin, development and migration to tissues. Mol Immunol. 2015; 63:9-17.

50. Jamur MC, Oliver C. Origin, maturation and recruitment of mast cell precursors. Front Biosci (Schol Ed). 2011; 3:1390-406.

51. Ehrlich P. Beitra"ge zur Theorie und Praxis der histologischen Fa"rbung: Leipzig University; 1878 .

52. Azizkhan RG. Mast cell heparin stimulates migration of capillary endothelial cells in vitro. J Exp Med. 1980; 152:931-44.

53. Cheng LE, Hartmann K, Roers A, Krummel MF, Locksley RM. Perivascular mast cells dynamically probe cutaneous blood vessels to capture immunoglobulin E. Immunity. 2013; 38:166-75. 
54. Wilhelm M, Silver R, Silverman AJ. Central nervous system neurons acquire mast cell products via transgranulation. Eur J Neurosci. 2005; 22:2238-48.

55. Voisin T, Bouvier A, Chiu IM. Neuro-immune interactions in allergic diseases: novel targets for therapeutics. Int Immunol. 2017; 29:247-61.

56. Yao W, Yang H, Yin N, Ding G. Mast Cell-Nerve Cell Interaction at Acupoint: Modeling Mechanotransduction Pathway Induced by Acupuncture. Int J Biol Sci. 2014; 10:511-9.

57. Poglio S, De Toni-Costes F, Arnaud E, Laharrague P, Espinosa E, Casteilla L, et al. Adipose tissue as a dedicated reservoir of functional mast cell progenitors. Stem Cells. 2010; 28:2065-72.

58. Koppert W, Blunk JA, Petersen LJ, Skov P, Rentsch K, Schmelz M. Different patterns of mast cell activation by muscle relaxants in human skin. Anesthesiology. 2001; 95:659-67.

59. Maurer M, Paus R, Czarnetzki BM. Mast cells as modulators of hair follicle cycling. Exp Dermatol. 1995; 4:266-71.

60. Kumamoto T, Shalhevet D, Matsue H, Mummert ME, Ward BR, Jester JV, et al. Hair follicles serve as local reservoirs of skin mast cell precursors. Blood. 2003; 102:1654-60.

61. Santos A, Lagares D. Matrix Stiffness: the Conductor of Organ Fibrosis. Curr Rheumatol Rep. 2018; 20:2.

62. Wickert LE, Pomerenke S, Mitchell I, Masters KS, Kreeger PK. Hierarchy of cellular decisions in collective behavior: Implications for wound healing. Sci Rep-Uk. 2016; 6.

63. Salgado CM, Silver RB, Bauer BS, Basu D, Schmitt L, Khakoo Y, et al. Skin of patients with large/giant congenital melanocytic nevi shows increased mast cells. Pediatr Dev Pathol. 2014; 17:198-203.

64. Rosin NL, Agabalyan N, Olsen K, Martufi G, Gabriel V, Biernaskie J, et al. Collagen structural alterations contribute to stiffening of tissue after split-thickness skin grafting. Wound Repair Regen. 2016; 24:263-74.

65. Akimoto S, Ishikawa O, Igarashi Y, Kurosawa M, Miyachi Y. Dermal mast cells in scleroderma: their skin density, tryptase/chymase phenotypes and degranulation. Br J Dermatol. 1998; 138:399-406.

66. Nishikori Y, Kakizoe E, Kobayashi Y, Shimoura K, Okunishi H, Dekio S. Skin mast cell promotion of matrix remodeling in burn wound healing in mice: relevance of chymase. Arch Dermatol Res. 1998; 290:553-60. 